\title{
Controlling Terahertz Surface Plasmon Properties on a Periodically Structured Silicon Surface
}

\author{
Gagan Kumar \\ Department of Physics, Indian Institute of Technology Guwahati, Guwahati, Assam 781039, India \\ Correspondence should be addressed to Gagan Kumar; gk@iitg.ernet.in
}

Received 31 October 2014; Revised 15 January 2015; Accepted 22 January 2015

Academic Editor: Alan C. Samuels

Copyright (C) 2015 Gagan Kumar. This is an open access article distributed under the Creative Commons Attribution License, which permits unrestricted use, distribution, and reproduction in any medium, provided the original work is properly cited.

\begin{abstract}
The paper presents experimental and numerical investigations on the terahertz (THz) surface plasmon propagation in a periodically patterned doped silicon substrate. Silicon substrates are periodically patterned with 2D array of vertical structures forming a plasmonic waveguide. The waveguide configurations are found to support resonant surface modes at certain frequencies which can occur anywhere depending on the structural parameters. The 2D pattern of vertical structures is observed to affect the $\mathrm{THz}$ surface plasmon propagation along the waveguide configuration. The periodicities are changed in both directions to examine the change in amplitude and cut-off frequencies of the resonant surface modes. The results are confirmed independently through finite element method based numerical simulations and compared with theory. The present study should find applications in facilitating the development of futuristic ultrahigh speed networks and devices operating at the terahertz frequencies.
\end{abstract}

\section{Introduction}

Recent years have witnessed significant developments in the field of plasmonic metamaterials. Metamaterials are the artificially designed subwavelength scale structures that have the ability to alter electromagnetic radiation propagation in a controlled fashion [1-7]. The artificially designed structures have been shown to guide and manipulate surface electromagnetic waves whose properties can be controlled with structural parameters $[8,9]$. The guided wave devices utilizing plasmonic metamaterials have led to several applications which include nanoimaging [10], nanoscale photonic components [11, 12], slow light systems [13], and biosensing [14-16]. There has been immense interest in developing guided wave components which can operate at terahertz frequencies. Terahertz frequency regime of the electromagnetic spectrum has the potential to significantly improve the efficiency and speed of the devices $[17,18]$. In this direction, plasmonic metamaterials have been investigated to play an important role, and guided wave components and devices operating at terahertz frequencies have been developed [1921]. The metals have been favorable choice for this purpose as they exhibit negligible dielectric and ohmic losses [22]. Williams et al. have demonstrated that a two-dimensional array of groove pattern with metal coating can support highly confined terahertz surface modes [23]. The metal coating is considerably thick (more than skin depth) to reduce the propagation losses. Zhu et al. also later on showed that when a thin metal sheet of stainless steel is periodically patterned with the one-dimensional array of apertures, then it supports high confined terahertz surface modes at certain frequencies which can be defined by the geometrical parameters of the apertures [24]. Subsequently, a significant amount of work has been noticed in this direction with different shapes of the metallic corrugations [25-29]. It is important to mention that these experimental investigations on guiding surface electromagnetic waves are inspired by Pendry's seminal work on plasmonic metamaterials [30, 31].

In spite of an extensive research work in the field of plasmonic metamaterials using metals, we have not been able to achieve active waveguide components such as modulators, ultrafast switches, filters, and active resonators. This is because of the difficulty in tuning the properties of the metal based waveguides. In order to overcome this issue, recently Kumar et al. demonstrate the planar plasmonic terahertz waveguides based on heavily doped silicon [32]. These waveguides can be more readily fabricated using photolithographic techniques in a clean room and properties 


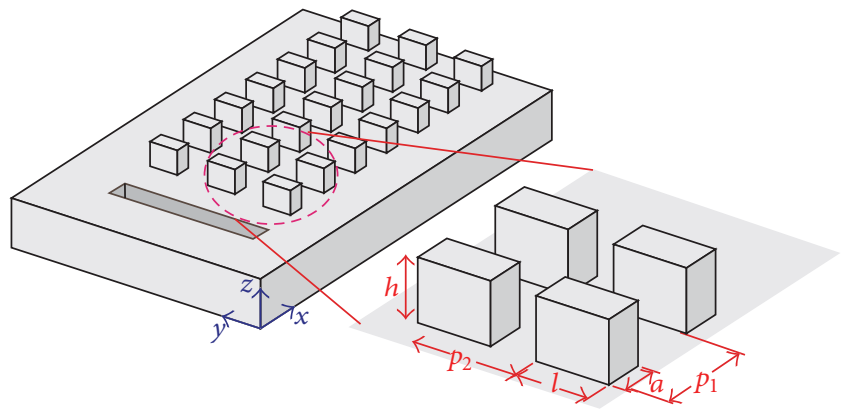

(a)

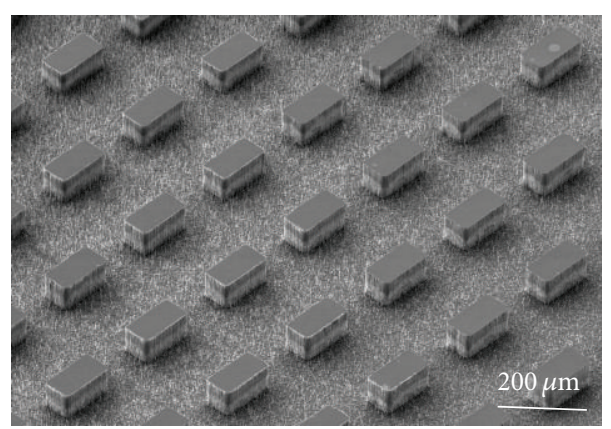

(b)

FIGURE 1: (a) Schematic of a heavily doped silicon surface structured with vertical rectangular structures. In the beginning, a rectangular shaped groove is designed to couple the incoming broadband terahertz radiation to the patterned surface. The inset shows a bigger view of a portion of the patterned waveguide with all the parameters defined. (b) It shows a scanning electron micrograph of a portion of the patterned waveguide comprising vertical structures.

of the substrates can be altered by changing the carrier concentration when externally stimulated, that is, by means of a laser or photo illumination. It has been found that a doped silicon can exhibit metallic properties when one reaches the dopant concentration of the order of $2.2 \times 10^{19} \mathrm{~cm}^{-3}$ [32, 33]. In assuming doped silicon with high conductivity, the classical Drude model approach is used under the free carrier approximation. It estimates dielectric constant values reasonably well, justifying our approximation. For instance, for the heavily doped silicon considered (dopant concentration $\left.\sim 10^{20} \mathrm{~cm}^{-3} ; \rho=2.5 \mathrm{~m} \Omega \mathrm{cm}\right)$, the model predicts a relative permittivity of $\varepsilon=(-1.3+2.0 i) \times 10^{4}$ at $1 \mathrm{THz}$, which is large enough to be accurately modeled as a perfect electric conductor. For comparison, the relative permittivity of $\mathrm{Al}$ at this frequency is calculated to be $\varepsilon=(-0.3+6.4 i) \times 10^{5}$. However, recent investigations have questioned its validity. It is believed that a modified model taking into account the electron-electron and electron-ion interactions may explain discrepancy [34]. In heavily doped silicon, one can possibly design and fabricate even complex geometries and accomplish plasmonic response [35]. In this direction, waveguides with geometries such as long V-shape structures [36] and concave pyramidal troughs have been demonstrated more recently [37]. Further, recently Kumar et al. demonstrated that a one-dimensional array of pillars forms a plasmonic waveguide and supports highly confined terahertz modes [32]. In certain applications such as modulators, sensors, and resonators, one may be required to have more interaction of the coupled $\mathrm{THz}$ wave with the resonant structures in order to achieve more sensitivity and responsivity; therefore, two- or more dimensional configurations could be effective. Further, a more pronounced resonant plasmonic behavior is anticipated in multidimensionally structured configurations. Therefore, a thorough investigation in this direction is prerequisite.

In this paper, the terahertz radiation propagation on a boron doped silicon surface structured with subwavelength scale square and rectangular shape vertical structures is examined both numerically and experimentally. The vertical structures are patterned in two dimensions and consequences of these structures on 1D terahertz surface plasmon propagation are investigated. A number of devices comprising vertical structures are fabricated with various different geometrical parameters. The $\mathrm{THz}$ time domain spectroscopy, a powerful tool for getting both amplitude and phase information, is used to characterize the $\mathrm{THz}$ transmission response. The patterned surfaces act as an effective media for the propagation of terahertz surface plasmons at a particular frequency. The antiresonant frequencies of the terahertz radiation modes are found to be dependent on the geometry of the structures and hence a desired frequency mode can be made to propagate by properly choosing the geometrical parameters of the structures. The effect of change of periodicity in both dimensions is examined numerically. The results are validated with a theoretical model. The paper is divided as follows. In Section 2, the fabrication of the samples and experimental setup, basically a modified $\mathrm{THz}$ time domain spectrometer used to characterize the samples, are discussed. In Section 3, experimental observations in conjunction with the numerical simulations are examined. This section also presents a detailed study on the behavior of antiresonant frequencies of the fundamental modes with geometrical parameters of the structures. The results are summarized in Section 4.

\section{Experimental Details}

The experimental part mainly consists of fabrication and characterization. The $2 \mathrm{D}$ vertical structures are fabricated on a heavily doped silicon substrate using advanced fabrication facilities. The silicon when heavily doped exhibits metallic properties making it a suitable candidate for the plasmonic guided wave applications at terahertz frequencies. The waveguide structures with subwavelength shape vertical rectangular structures are fabricated on a highly $p$-doped silicon surface (carrier density $\sim 5 \times 10^{20} \mathrm{~cm}^{-3}$ ) on an area of $5 \mathrm{~cm} \times 4 \mathrm{~mm}$. The photo lithographically patterned silicon surfaces are etched through deep reactive ion etching (DRIE) with an etch rate of $1.5 \mu \mathrm{m} / \mathrm{s}$. The schematic diagram of a patterned waveguide is shown in Figure 1(a). The inset shows 


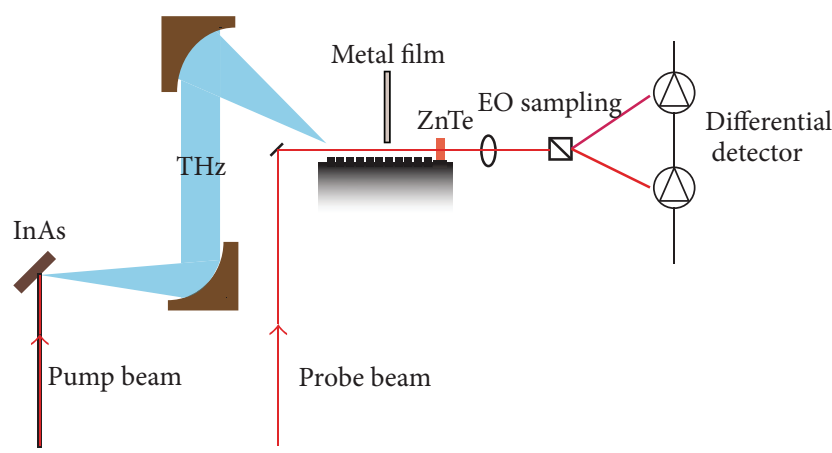

FIgure 2: Schematic of the experimental setup. The InAs crystal generates $\mathrm{THz}$ through Photo-Dember effect. The detection is done via electrooptic sampling. The (110) ZnTe crystal is used for the detection of the THz.

a bigger view of the pattern with all the parameters being clearly defined. In the figure, " $l$ " is the length, " $a$ " is the width, " $h$ " is the height, and $p_{1}$ and $p_{2}$ stand for the periodicity of the vertical structures in $x$ - and $y$-directions, respectively. A scanning electron micrograph (SEM) of a portion of a fabricated waveguide pattern is shown in Figure 1(b). At one end of the patterned surface, a rectangular groove is fabricated in a second step of photolithography. The rectangular groove is patterned with a width to length $(a \times l)$ ratio of $300 \mu \mathrm{m} \times 2.5 \mathrm{~mm}$. The groove is again etched using deep reactive ion etching to a depth of $100 \mu \mathrm{m}$. The structured waveguide patterns for their plasmonic response are characterized using modified $\mathrm{THz}$ time domain spectroscopy setup. The schematic of the experimental setup is shown in Figure 2. An optical beam at $800 \mathrm{~nm}$ wavelength from the amplified laser system is split into $80: 20$ in power for use as pump and probe beam, respectively. The femtosecond pulsed beam from the amplified laser system with average power of $\sim 1.2 \mathrm{~W}$, repetition rate of $1 \mathrm{kHz}$, and temporal pulse duration of $\sim 43 \mathrm{fs}$ is used for the generation of terahertz through a $1 \mathrm{~mm}$ thick InAs via Photo-Dember effect. The s-polarized pump beam incident on a nonlinear crystal leads to broadband terahertz radiation generation which is collected and collimated using off-axis parabolic mirrors. The patterned substrate in the setup is placed in such a way that the terahertz beam from the second parabolic mirror focuses on the coupling groove fabricated at one end of the structured waveguide. The spot size of the $\mathrm{THz}$ beam focusing on coupling groove is approximately $3 \mathrm{~mm}$. By hitting free space $\mathrm{THz}$ at an angle, one may anticipate a free space signal arriving at the detection crystal which can interfere with the surface plasmons. In order to minimize this, we used a metal film in the middle of waveguide held at a gap of $\sim 2 \mathrm{~mm}$ above the surface as shown in Figure 2. One may always use more promising approaches to avoid unsought free space signal. This includes a curved sheet for separating free space $\mathrm{THz}$ from the terahertz surface plasmons as proposed by Jeon and Grischkowsky [38]. The incidence of input terahertz at $90^{\circ}$ with respect to coupling groove can also help in minimizing the unsought $\mathrm{THz}$ signal arriving at the detection crystal [37]. The terahertz wave once coupled to the waveguide has the electric field component pointing normal to the surface and magnetic field along the surface in the $y$-direction. The terahertz surface plasmon polaritons (SPPs) after propagating along the patterned structures are detected by a (110) ZnTe crystal of thickness $1 \mathrm{~mm}$ with crystal face oriented orthogonal to the $x$-axis. We used the electrooptic sampling method to measure the specific vector component of the SPPs. The detection system utilizes differential detection for better sensitivity.

\section{Results and Discussions}

In Figure 3, both the measured and simulated waveguide transmission spectra are shown for the vertical subwavelength scale structures designed across the $5 \mathrm{~cm} \times 4 \mathrm{~mm}$ silicon substrate. The experimental results are obtained using THz-TDS spectroscopy discussed above. The corresponding structures are numerically simulated using finite element method based electromagnetic simulation package, that is, CST Microwave Studio. Figure 3(a) shows the frequency domain spectra corresponding to the patterned vertical rectangular structures having parameters: length " $l$ " $=300 \mu \mathrm{m}$, width " $a$ " $=200 \mu \mathrm{m}$, lattice constant " $p_{1} "=" p_{2} "=400 \mu \mathrm{m}$, and height " $h$ " $=100 \mu \mathrm{m}$. The blue traces correspond to the experiment, while green traces correspond to the simulation. A discrete $\mathrm{THz}$ source was used at one end of the waveguide for simulation and probe detector was placed at the other end. The results from both the simulation and experiment are in fairly good agreement and are found to support resonant modes. Both spectra do show a resonant mode at $0.36 \mathrm{THz}$, which we call as the fundamental plasmonic mode supported by the structured substrate. The experiment does show a higher order mode as well at $1 \mathrm{THz}$ frequency which is missing in the simulated spectra. Further, it is noted that experimental spectral features are broader than the simulated resonant features. It is important to mention that, in simulations, highly doped silicon is considered as perfect electrical conductor (PEC) which is a good approximation at $\mathrm{THz}$ frequencies; however, in practice, the assumption may slightly differ, resulting in broader spectral features in the experiment. Further, geometrical defects and greater losses, namely, scattering, diffraction, and so forth, associated with the fabricated structures, add to the discrepancies. One may also see that, on the higher frequency side, experimental observations do not comply with the simulation results. This is believed to be due to the inaccuracy in the simulation at the shorter wavelengths resulting from the smaller mesh size which is of the order of $p / 10$. Further, greater quantization errors are anticipated at the higher frequency side that is beyond the first Brillouin boundary which is given by $c / 2 p$. In our case, this is calculated to be $0.38 \mathrm{THz}$. The experimental and simulation observations were further verified theoretically. For the fundamental TM-like mode, neglecting the diffraction effect under the assumption of $\lambda \gg p$, the dispersion relation is given by [31]

$$
k_{x}=k_{0}\left(1+\left(\frac{a}{p}\right)^{2} \tan ^{2}\left(k_{0} h\right)\right)^{1 / 2}
$$




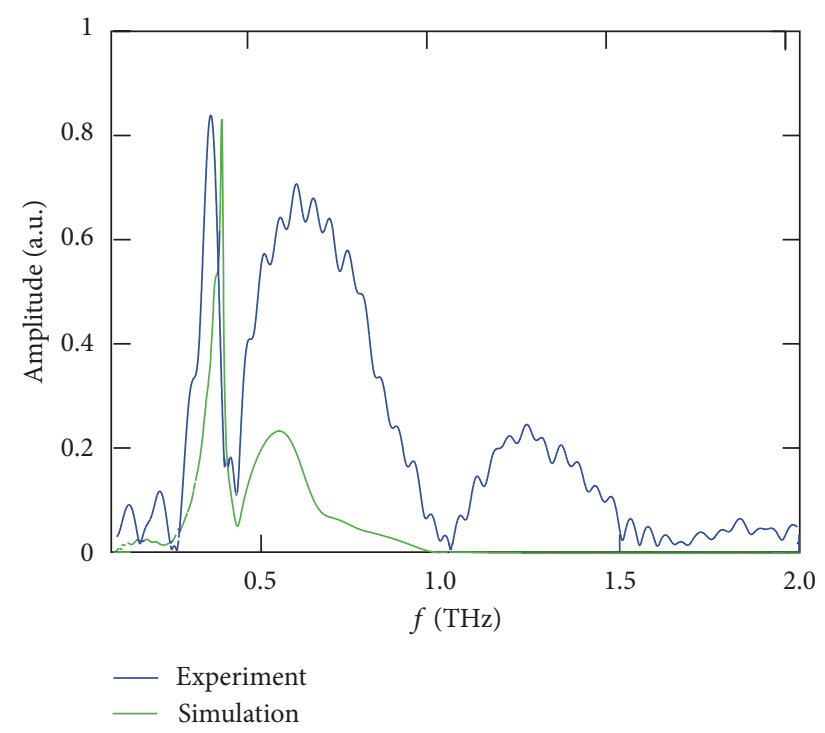

(a)

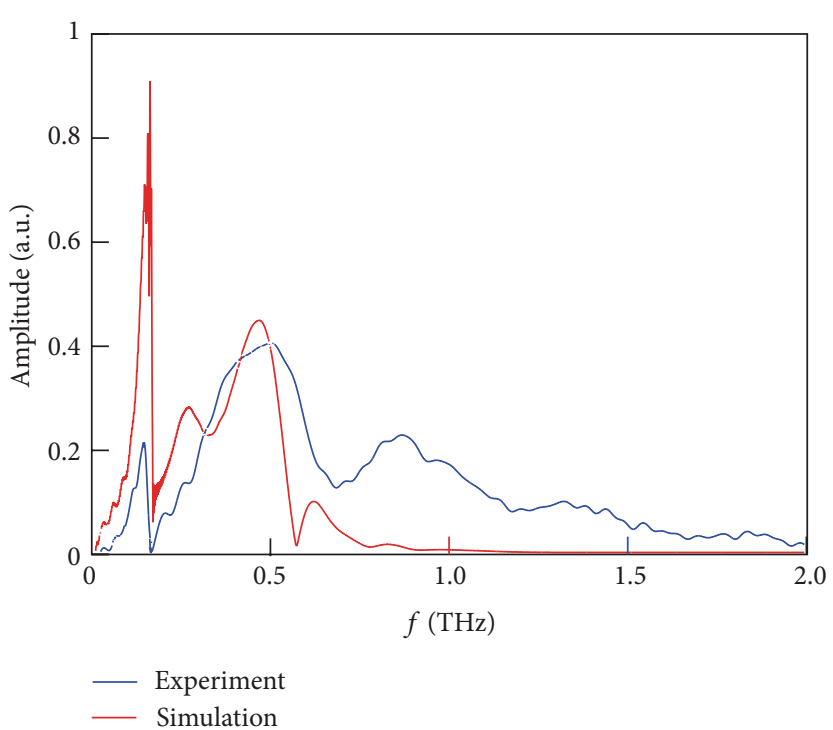

(b)

FIGURE 3: Amplitude transmission across the silicon substrate patterned with subwavelength scale vertical rectangular structures. (a) Measured and numerically simulated frequency domain spectra for the parameters: length "l" $=300 \mu \mathrm{m}$, width " $a$ " $=200 \mu \mathrm{m}$, lattice constant " $p_{1} "=" p_{2} "=400 \mu \mathrm{m}$, and height " $h "=100 \mu \mathrm{m}$. (b) Frequency domain spectra correspond to the parameters: "l" $=300 \mu \mathrm{m}$, " $a "=200 \mu \mathrm{m}$, " $p_{1} "=" p_{2} "=400 \mu \mathrm{m}$, and height " $h "=300 \mu \mathrm{m}$.

where $k_{x}$ is the wave number in the direction of propagation. $k_{0}=\omega / c$ is the free space wave number, $h$ is the height of the pillars, and $a / p$ is the ratio of width to periodicity for the vertical structures. We calculated the antiresonant frequency at $k_{x} p / \pi=1$ for the "l" $=300 \mu \mathrm{m}, " a "=200 \mu \mathrm{m}$, " $p$ " = " $p_{1}$ " = $400 \mu \mathrm{m}$, and " $h "=100 \mu \mathrm{m}$ and it turned out to be $0.36 \mathrm{THz}$, which is same as the observed antiresonant frequency of the fundamental mode. Later in this paper, we will see that a variation of periodicity " $p_{2}$ " does not affect the fundamental resonant frequency and hence the analytical modal given by (1) accurately predicts the frequency of the fundamental terahertz mode.

In Figure 3(b), another patterned configuration of vertical rectangular structures with a height of " $h$ " $=300 \mu \mathrm{m}$ is examined. Other parameters of the structured substrate are same as the previous pattern. Again, it is apparent from the figure that both the experimental and simulation spectra exhibit resonant behavior and antiresonant frequencies of the fundamental mode are found to be in good agreement. The blue traces correspond to the experiment; however, red traces represent numerically simulated frequency domain spectra. The antiresonant frequency of the fundamental mode is observed to be $0.19 \mathrm{THz}$. One may see the higher order modes as well in the figure which are more pronounced in case of numerical simulation. The discrepancy in the experiment and simulation on the higher frequency side can be understood again from the explanation given for earlier plot.

In Figure 4(a), the effect of variation of height " $h$ " of the vertical square shape structures on the terahertz surface plasmon propagation is examined numerically. The figure shows the numerically calculated frequency domain spectra for the periodically patterned structured surface for the different heights of the vertical structures. The periodicities $p_{1}$ and $p_{2}$ are the same, that is, $400 \mu \mathrm{m}$ in both directions. The other parameters are " $l "=200 \mu \mathrm{m}$ and " $a$ " = $200 \mu \mathrm{m}$. The red traces in the figure correspond to the $h=$ $100 \mu \mathrm{m}$, while blue, green, and brown color traces represent frequency domain spectra corresponding to the vertical structures of $h=200 \mu \mathrm{m}, 300 \mu \mathrm{m}$, and $400 \mu \mathrm{m}$, respectively. One may note that the amplitude of the waveguide transmission decreases as the height of the structures increases. Hence, one is required to fabricate structures with shorter height for the efficient transmission; however, frequency should be kept in mind. Further, it may be noted that as the height increases the antiresonance frequency goes down. It can be explained from (1) which clearly shows a dependence of the dispersion relation on the height of the structures. The frequency of saturation in the dispersion relation can be approximated with antiresonant frequency of the fundamental mode which changes with height. One may also note that waveguide geometries with taller structures would result in lower group velocity of the fundamental modes and the lower cut-off frequencies. Therefore, one can design 2D plasmonic waveguides keeping in mind the amplitude as well as the frequency of the terahertz waves. In Figure 4(b), an electric field profile across the structured surface in the $X Y$ plane is shown at $0.36 \mathrm{THz}$. One may note that there is a strong field confinement along the vertical square shape structures at the resonance. The field profile at the nonresonant frequency was also examined (not shown here) and no confinement along the surface was seen. In Figures 5(a) and 5(b), terahertz amplitude transmissions for a patterned substrate with different periodicity in the $x$ - and $y$ directions are shown. Unlike the groove patterns as examined 


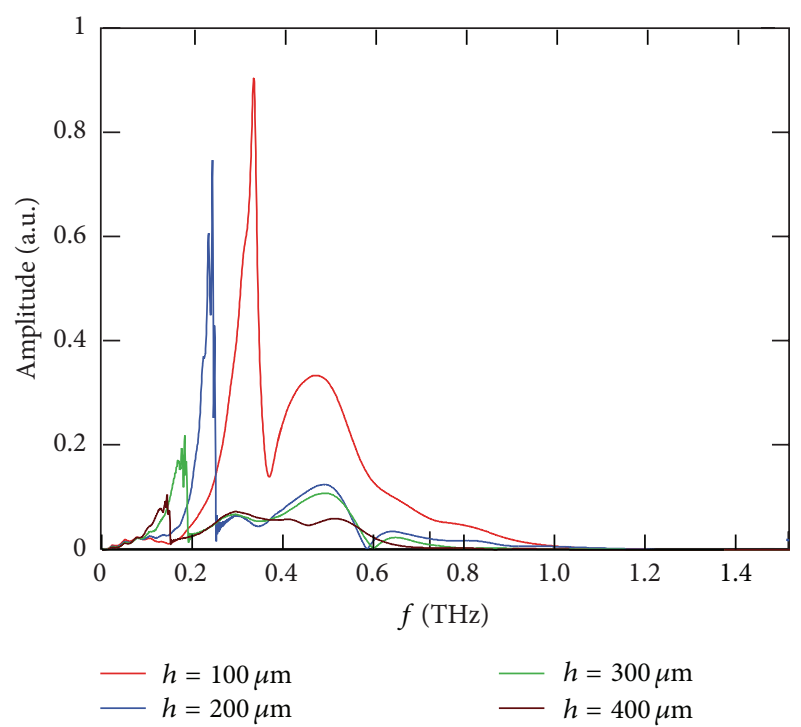

(a)

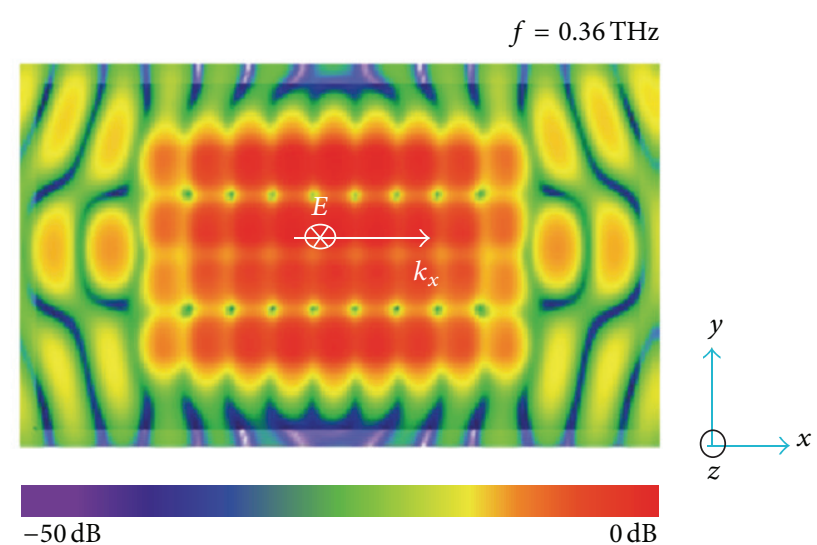

(b)

Figure 4: (a) Numerically examined frequency domain spectra for the four patterned substrates with different heights from $h=100 \mu \mathrm{m}$ to $h=400 \mu \mathrm{m}$ of the vertical square shape structures. The other parameters are "l" $=200 \mu \mathrm{m}, " a "=200 \mu \mathrm{m}$, and " $p_{1} "=" p_{2} "=400 \mu \mathrm{m}$. (b) The electric field profile in the $X Y$ plane at $0.36 \mathrm{THz}$ depicts a strong confinement at this frequency. " $E$ " points to the electric field normal to the surface while $k_{x}$ points to the direction of propagation of terahertz surface plasmons.

in the literature in the past [25], the periodicity strongly influences the resonant mode propagation in the $x$-direction as shown in Figure 5(a). Figure 5(a) shows red, green, and blue trances which correspond to the $p_{1}=300 \mu \mathrm{m}, 400 \mu \mathrm{m}$, and $500 \mu \mathrm{m}$, respectively. The other parameters of the structures are "l" $=200 \mu \mathrm{m}, " a "=200 \mu \mathrm{m}$, and " $p_{2} "=400 \mu \mathrm{m}$. With smaller periodicity, the coupling between the structures is stronger and one see a sharp resonance as can be seen in the red color. When the periodicity increases, coupling between successive structures becomes weaker and resonant effect is not pronounced. One may observe a broadband response of the terahertz radiation propagation with periodicity on the order of wavelength scale. However, the amplitude transmission is very small in that case and there is poor confinement for the terahertz radiations. In Figure 5(b), effect of periodicity variation in the $y$-direction is examined on the resonant behavior of the patterned surface. It is observed that the fundamental resonant frequency is not influenced by the periodicity in this case. One may certainly observe a change in the amplitude of the fundamental resonance with a change in the periodicity. As periodicity is increased, the amplitude goes down. This is because of the weak coupling between the structures and hence lower contribution to the surface plasmon propagation in the $x$-direction from the structures in the $y$-direction. The antiresonant frequencies are caused by the Fano interference of discrete and continuum spectrums. The TM mode propagation in the $x$-direction and associated cut-off frequency are dependent on the periodicity in the $x$-direction as can be noticed from (1). For THz surface plasmons, field in the $y$-direction is negligible and periodicity is not expected to change the antiresonant frequency. Further, the effect of the lateral length (in $y$-direction), that is, "l," and the width " $a$ " of the structures on the propagation properties of the terahertz radiation propagation is investigated. The numerically examined results are shown in Figure 5(c). The red triangular dots represent the numerically calculated antiresonant frequencies for different values of the width " $a$ " of the vertical square shape structures. The other parameters of the structures are "l" $=200 \mu \mathrm{m}$, height " $h "=100 \mu \mathrm{m}$, and " $p_{1} "=" p_{2} "=400 \mu \mathrm{m}$. The calculated data points are fitted with the straight line equation which is represented in the red color. One may note that straight line fits fairly well with the data implying that a variation in the width does not leave any effect on the antiresonant frequency of the fundamental mode. Similarly, the effect of length is examined in the same figure. The blue circular dots represent the antiresonant frequencies of the fundamental mode for various different lengths of the rectangular structures. The width and other parameters are kept constant in this case and are taken as " $a "=200 \mu \mathrm{m}$, height " $h "=85 \mu \mathrm{m}$, and " $p_{1} "=$ " $p_{2} "=400 \mu \mathrm{m}$. The data points are again fitted with the straight line equation which is represented by the blue line. Again it fits the data very well and hence one can infer that change in the length of the vertical structures does not leave any effect on the antiresonant frequency of the fundamental mode. In Figure 5(d), a comparison between the transverse confinement of $\mathrm{THz}$ surface plasmon modes in $1 \mathrm{D}$ and 2D structured waveguides is shown for parameters: "l" $=200 \mu \mathrm{m}, " a "=200 \mu \mathrm{m}$, height " $h "=100 \mu \mathrm{m}$, and " $p_{1} "=$ " $p_{2} "=400 \mu \mathrm{m}$. Using 3D numerical simulation software, the field was measured in the transverse direction $(z$-axis) in the middle of the waveguides using discrete detection ports. The data points are exponentially fitted. In case of $1 \mathrm{D}$ sample, the $1 / e$ decay length was observed $\sim 420 \mu \mathrm{m}$, while in $2 \mathrm{D}$ sample it came out $\sim 550 \mu \mathrm{m}$. Therefore, transverse confinement of the terahertz surface plasmon decreases when waveguide is 


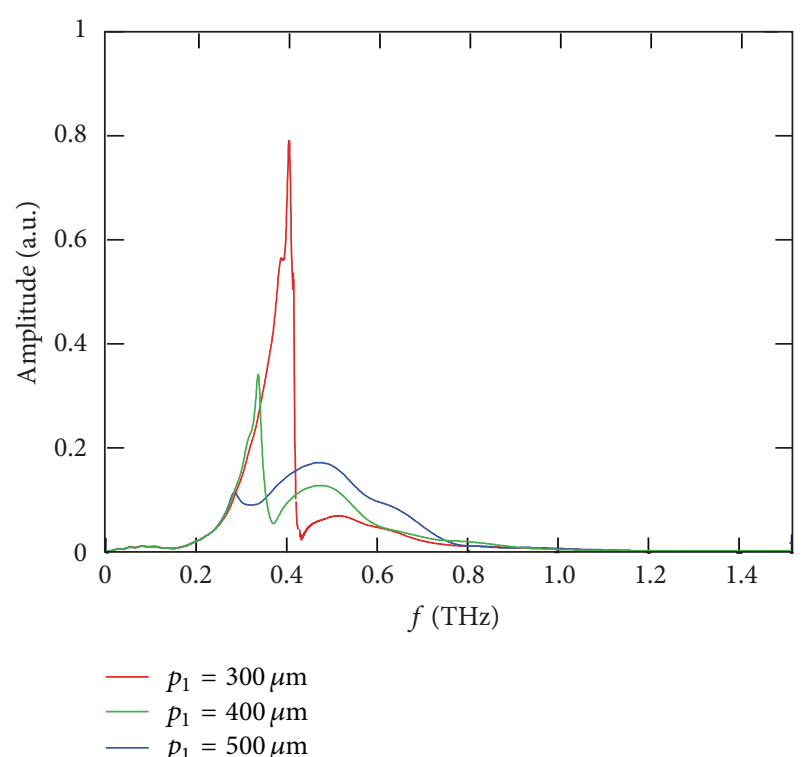

(a)

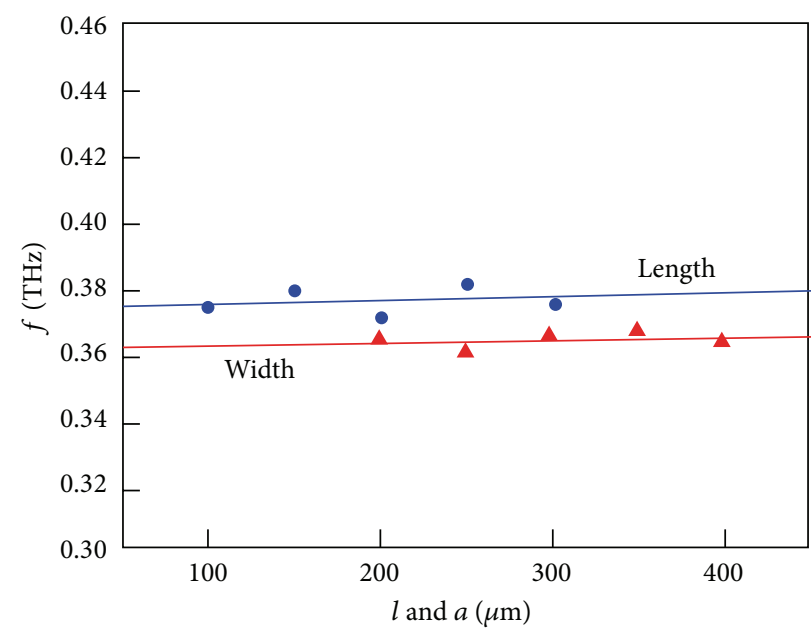

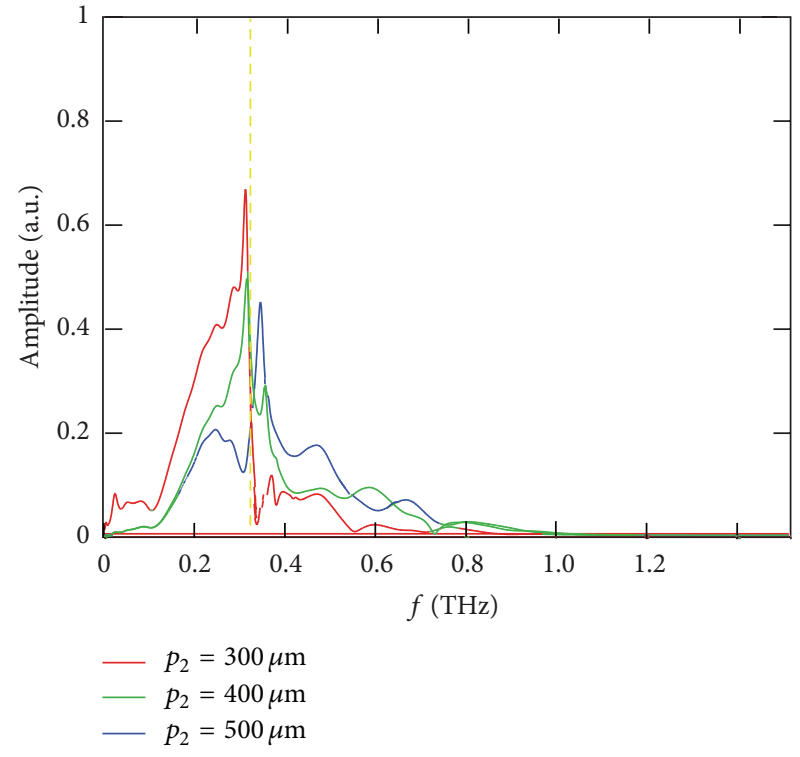

(b)

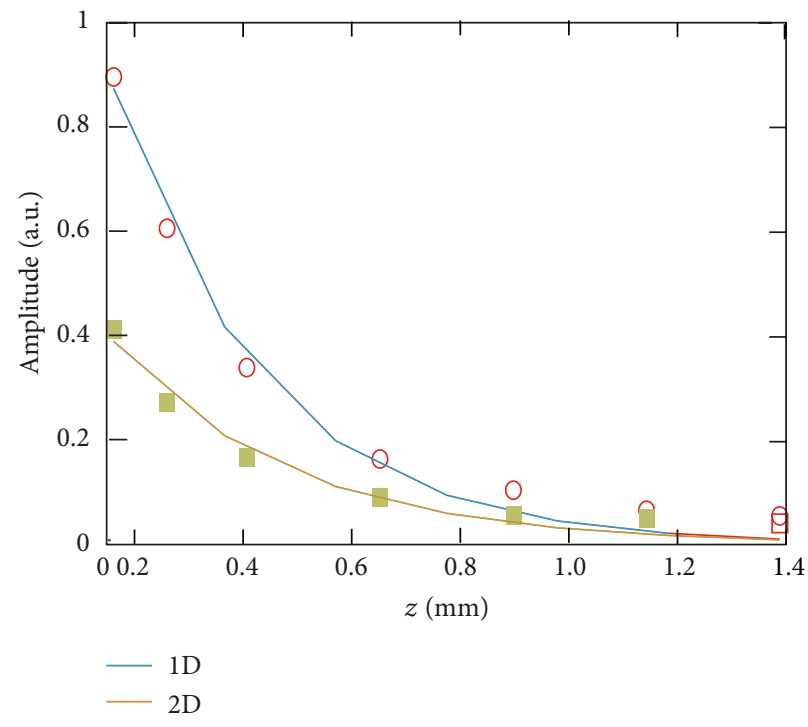

(d)

FIGURE 5: (a) Numerically examined frequency domain spectra for three patterned substrates with different periodicity in the $x$-direction, however constant periodicity in the $y$-direction; that is, " $p_{2} "=400 \mu \mathrm{m}$. The other parameters are "l" $=200 \mu \mathrm{m}$, " $a$ " $=200 \mu \mathrm{m}$, and " $h$ " $=$ $100 \mu \mathrm{m}$. (b) Frequency domain spectra for three patterned substrates with same $p_{1}=400 \mu \mathrm{m}$, however different values of $p_{2}$. The other parameters are same as those in (a). (c) It shows the variation of antiresonant frequency with length and width of the vertical structures. The calculated values are fitted with straight lines. (d) shows a comparison of the transverse confinement between 1D and 2D structured samples.

patterned in both spatial directions. The information can be used to design the plasmonic metamaterials based terahertz active and passive components and can prove to be the building blocks in developing the terahertz networks.

\section{Conclusions}

In this paper, 2D periodically patterned vertical structures on a silicon substrate are examined for terahertz surface plasmon polariton propagation. Heavily doped silicon substrates are periodically patterned with $2 \mathrm{D}$ array of structures resulting in plasmonic waveguide configurations. The experimental measurements through a $\mathrm{THz}$ time domain spectrometer show resonant terahertz mode propagation at certain frequencies defined by the geometrical parameters of the structures. Numerically simulated structures with similar shape and sizes yield results which are consistent with the experimental observations. An increase in the height of the structures results in a decrease in the amplitude as well as antiresonant frequency. The alterations in width and length of the structures do not affect antiresonant behavior. In order to understand the effect of structures in $2 \mathrm{D}$ on the 
$\mathrm{THz}$ surface plasmon propagation properties, periodicity is changed in both spatial directions and resonant behavior is examined. An alteration in periodicity in $x$-direction significantly affects the frequency and amplitude of the resonant mode; however, cut-off frequency remains unchanged in other spatial directions. Further, a comparison in the transverse confinement of the $1 \mathrm{D}$ and $2 \mathrm{D}$ structured samples is made. The $1 / e$ decay length of the $\mathrm{THz}$ surface plasmons increases under $2 \mathrm{D}$ patterning signifying a decrease in the transverse confinement. The study is anticipated to greatly felicitate the designing of futuristic devices and networks on silicon surfaces capable of operating at terahertz frequencies.

\section{Conflict of Interests}

The author declares that there is no conflict of interests regarding the publication of this paper.

\section{Acknowledgment}

The author would like to thank the University of Maryland for the characterization and fabrication facilities.

\section{References}

[1] N. Engheta and R. W. Ziolkowski, Metamaterials: Physics and Engineering Explorations, John Wiley \& Sons, New York, NY, USA, 2006.

[2] J. Hao, J. Wang, X. Liu, W. J. Padilla, L. Zhou, and M. Qiu, "High performance optical absorber based on a plasmonic metamaterial," Applied Physics Letters, vol. 96, no. 25, Article ID 251104, 2010.

[3] A. Christ, O. J. F. Martin, Y. Ekinci, N. A. Gippius, and S. G. Tikhodeev, "Symmetry breaking in a plasmonic metamaterial at optical wavelength," Nano Letters, vol. 8, no. 8, pp. 2171-2175, 2008.

[4] D. F. P. Pile and D. K. Gramotnev, "Plasmonic sub wavelength waveguides: next to zero losses at sharp bends," Optics Letters, vol. 30, no. 10, pp. 1186-1188, 2005.

[5] Y. Zhao and A. Alù, "Manipulating light polarization with ultrathin plasmonic metasurfaces," Physical Review B-Condensed Matter and Materials Physics, vol. 84, no. 20, Article ID 205428, 2011.

[6] M. Navarro-Cía, M. Beruete, S. Agrafiotis, F. Falcone, M. Sorolla, and S. A. Maier, "Broadband spoof plasmons and subwavelength electromagnetic energy confinement on ultrathin metafilms," Optics Express, vol. 17, no. 20, pp. 18184-18195, 2009.

[7] D. R. Smith, J. B. Pendry, and M. C. K. Wiltshire, "Metamaterials and negative refractive index," Science, vol. 305, no. 5685, pp. 788-792, 2004.

[8] S. A. Maier, Plasmonics: Fundamentals and Applications: Fundamentals and Applications, Springer, 2007.

[9] J. A. Schuller, E. S. Barnard, W. Cai, Y. C. Jun, J. S. White, and M. L. Brongersma, "Plasmonics for extreme light concentration and manipulation," Nature Materials, vol. 9, no. 3, pp. 193-204, 2010.

[10] S. Kawata, Y. Inouye, and P. Verma, "Plasmonics for near-field nano-imaging and superlensing," Nature Photonics, vol. 3, no. 7, pp. 388-394, 2009.
[11] S. I. Bozhevolnyi, "Plasmonic nano-guides and circuits," in Proceedings of the Plasmonics and Metamaterials (META PLAS '08), Optical Society of America, October 2008.

[12] N. Engheta, "Circuits with light at nanoscales: optical nanocircuits inspired by metamaterials," Science, vol. 317, no. 5845, pp. 1698-1702, 2007.

[13] Q. Gan, Z. Fu, Y. J. Ding, and F. J. Bartoli, “Ultrawide-bandwidth slow-light system based on $\mathrm{THz}$ plasmonic graded metallic grating structures," Physical Review Letters, vol. 100, no. 25, Article ID 256803, 2008.

[14] J. N. Anker, W. P. Hall, O. Lyandres, N. C. Shah, J. Zhao, and R. P. Van Duyne, "Biosensing with plasmonic nanosensors," Nature Materials, vol. 7, no. 6, pp. 442-453, 2008.

[15] G. Kumar, D. B. Singh, and V. K. Tripathi, "Surface enhanced Raman scattering of a surface plasma wave," Journal of Physics D: Applied Physics, vol. 39, no. 20, pp. 4436-4439, 2006.

[16] A. V. Kabashin, P. Evans, S. Pastkovsky et al., "Plasmonic nanorod metamaterials for biosensing," Nature Materials, vol. 8, no. 11, pp. 867-871, 2009.

[17] M. Tonouchi, "Cutting-edge terahertz technology," Nature Photonics, vol. 1, no. 2, pp. 97-105, 2007.

[18] P. H. Siegel, "Terahertz technology," IEEE Transactions on Microwave Theory and Techniques, vol. 50, no. 3, pp. 910-928, 2002.

[19] D. Wu, N. Fang, C. Sun et al., "Terahertz plasmonic high pass filter," Applied Physics Letters, vol. 83, no. 1, pp. 201-203, 2003.

[20] N. Yu, Q. J. Wang, M. A. Kats et al., "Designer spoof surface plasmon structures collimate terahertz laser beams," Nature Materials, vol. 9, no. 9, pp. 730-735, 2010.

[21] G. Kumar, A. Cui, S. Pandey, and A. Nahata, "Planar terahertz waveguides based on complementary split ring resonators," Optics Express, vol. 19, no. 2, pp. 1072-1080, 2011.

[22] S. A. Maier, S. R. Andrews, L. Martín-Moreno, and F. J. GarcíaVidal, "Terahertz surface plasmon-polariton propagation and focusing on periodically corrugated metal wires," Physical Review Letters, vol. 97, no. 17, Article ID 176805, 2006.

[23] C. R. Williams, S. R. Andrews, S. A. Maier, A. I. FernándezDomínguez, L. Martín-Moreno, and F. J. García-Vidal, "Highly confined guiding of terahertz surface plasmon polaritons on structured metal surfaces," Nature Photonics, vol. 2, no. 3, pp. 175-179, 2008.

[24] W. Zhu, A. Agrawal, and A. Nahata, "Planar plasmonic terahertz guided-wave devices," Optics Express, vol. 16, no. 9, pp. 62166226, 2008.

[25] G. Kumar, S. Pandey, A. Cui, and A. Nahata, "Planar plasmonic terahertz waveguides based on periodically corrugated metal films," New Journal of Physics, vol. 13, no. 3, Article ID 033024, 2011.

[26] S. P. Burgos, H. W. Lee, E. Feigenbaum, R. M. Briggs, and H. A. Atwater, "Synthesis and characterization of plasmonic resonant guided wave networks," Nano letters, vol. 14, no. 6, pp. 32843292, 2014.

[27] S. R. Andrews, "Microstructured terahertz waveguides," Journal of Physics D: Applied Physics, vol. 47, no. 37, Article ID 374004, 2014.

[28] W. Zhu, A. Agrawal, A. Cui, G. Kumar, and A. Nahata, "Engineering the propagation properties of planar plasmonic terahertz Waveguides," IEEE Journal on Selected Topics in Quantum Electronics, vol. 17, no. 1, pp. 146-153, 2011.

[29] J. J. Wood, L. A. Tomlinson, O. Hess, S. A. Maier, and A. I. Fernández-Domínguez, "Spoof plasmon polaritons in slanted 
geometries," Physical Review B: Condensed Matter and Materials Physics, vol. 85, no. 7, Article ID 075441, 2012.

[30] J. B. Pendry, L. Martín-Moreno, and F. J. Garcia-Vidal, "Mimicking surface plasmons with structured surfaces," Science, vol. 305, no. 5685, pp. 847-848, 2004.

[31] F. J. Garcia-Vidal, L. Martín-Moreno, and J. B. Pendry, "Surfaces with holes in them: new plasmonic metamaterials," Journal of Optics A: Pure and Applied Optics, vol. 7, no. 2, pp. S97-S101, 2005.

[32] G. Kumar, S. Li, M. M. Jadidi, and T. E. Murphy, "Terahertz surface plasmon waveguide based on a one-dimensional array of silicon pillars," New Journal of Physics, vol. 15, Article ID 085031, 2013.

[33] H. Seidel, L. Csepregi, A. Heuberger, and H. Baumgaertel, "Anisotropic etching of crystalline silicon in alkaline solutions. I. Orientation dependence and behavior of passivation layers," Journal of the Electrochemical Society, vol. 137, no. 11, pp. 36123626, 1990.

[34] S. Pandey, S. Liu, B. Gupta, and A. Nahata, "Self-referenced measurements of the dielectric properties of metals using terahertz time-domain spectroscopy via the excitation of surface plasmon-polaritons," Photonics Research, vol. 1, no. 4, pp. 148153, 2013.

[35] A. K. Azad, Y. Zhao, and W. Zhang, “Transmission properties of terahertz pulses through an ultrathin subwavelength silicon hole array," Applied Physics Letters, vol. 86, no. 14, Article ID 141102, pp. 1-3, 2005.

[36] S. Li, M. M. Jadidi, T. E. Murphy, and G. Kumar, “Terahertz surface plasmon polaritons on a semiconductor surface structured with periodic V-grooves," Optics Express, vol. 21, no. 6, pp. 70417049, 2013.

[37] S. Li, M. M. Jadidi, T. E. Murphy, and G. Kumar, "Plasmonic terahertz waveguide based on anisotropically etched silicon substrate," IEEE Transactions on Terahertz Science and Technology, vol. 4, no. 4, pp. 454-458, 2014.

[38] T.-I. Jeon and D. Grischkowsky, "THz Zenneck surface wave (THz surface plasmon) propagation on a metal sheet," Applied Physics Letters, vol. 88, no. 6, Article ID 061113, 2006. 

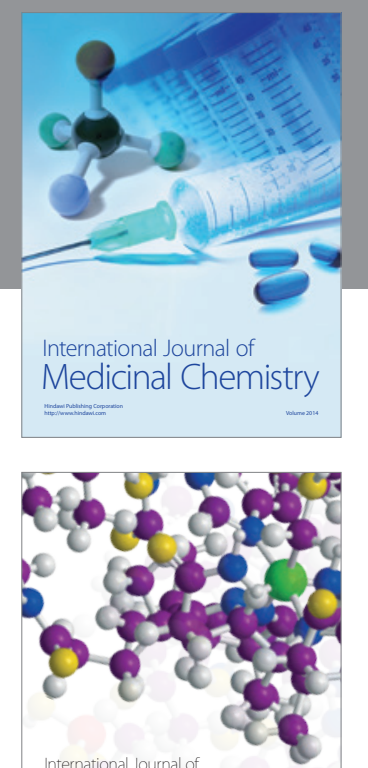

\section{Carbohydrate} Chemistry

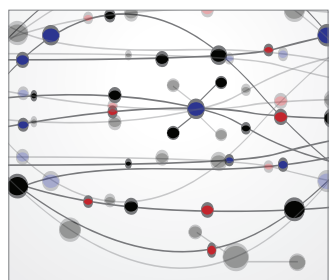

The Scientific World Journal
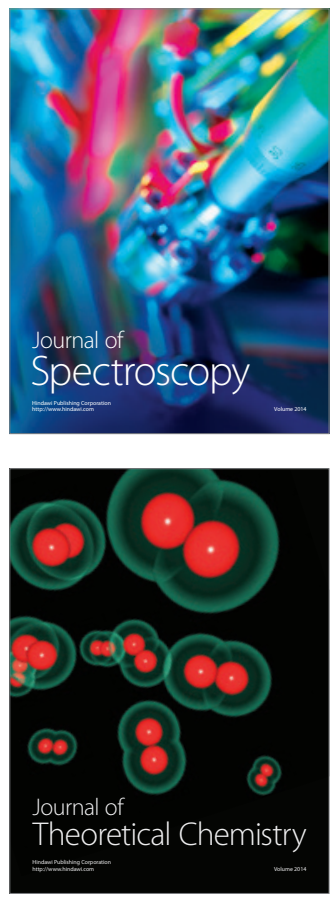
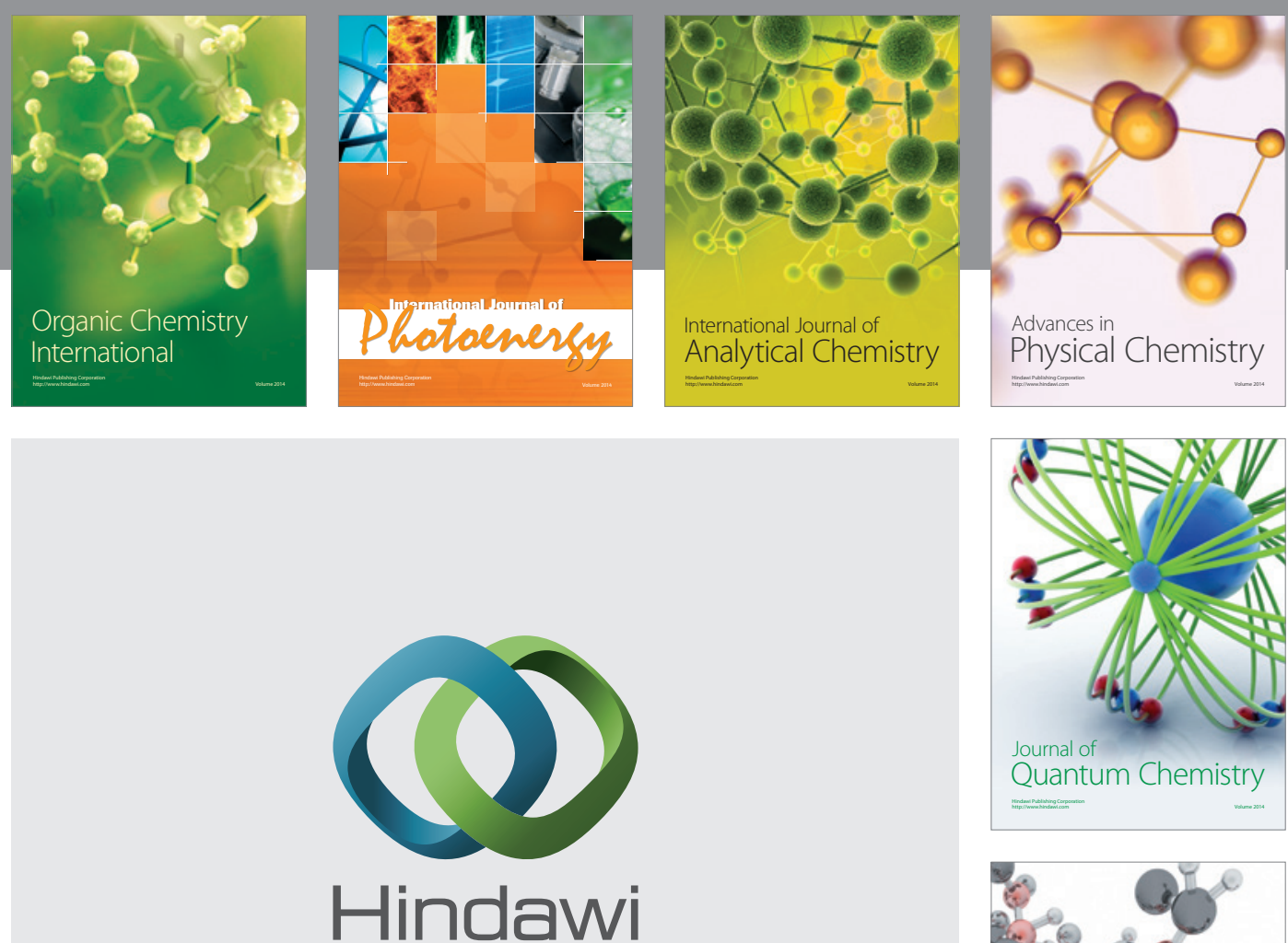

Submit your manuscripts at

http://www.hindawi.com

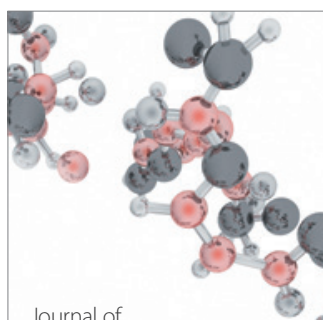

Analytical Methods

in Chemistry

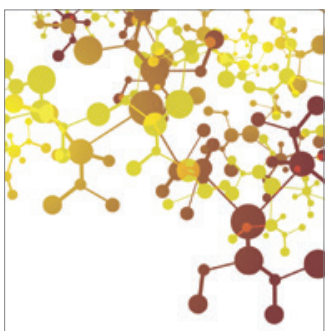

Journal of

Applied Chemistry

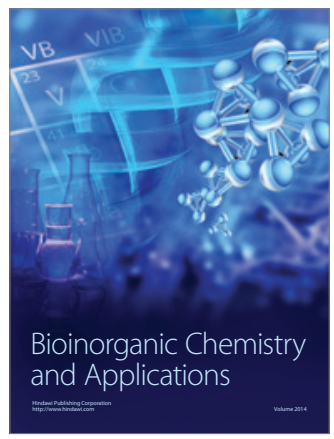

Inorganic Chemistry
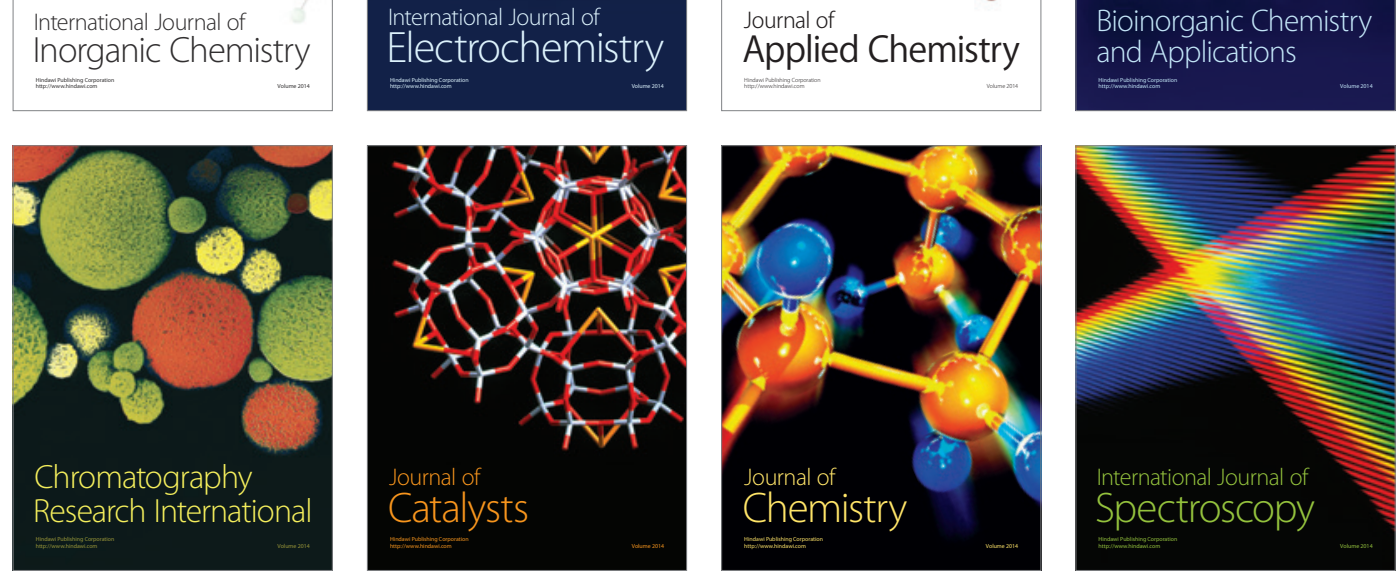